\title{
QUALITY ASSESSMENT OF FRIED POTATO WEDGES BY FUZZY LOGIC AND TEXTURE ANALYSES
}

\author{
P. K. Ghosh and P. BhattacharjeE* \\ Department of Food Technology and Biochemical Engineering, Jadavpur University, Kolkata 700032. India
}

(Received: 25 September 2012; accepted: 8 July 2013)

\begin{abstract}
Potato wedges were deep, shallow, and par-fried in soybean, sesame, and mustard oils using a laboratory formulated citric acid based antioxidant. The wedges were stored at $-18{ }^{\circ} \mathrm{C}$ for $10,20,30$, and 40 days. The quality of the wedges was assessed by subjective method (sensory analysis) and objective method (texture studies). Fuzzy logic was applied as a tool to analyze the linguistic data of subjective evaluation. Objective evaluation was conducted by studies on hardness, shear modulus and shear energy of the wedges. The wedges with highest defuzzified scores (obtained by sensory evaluation) and minimum changes in textural properties $(\mathrm{P}<0.05)$ were obtained in par-fried soybean oil (after 40 days storage) and in deep fried sesame and mustard oils (both after 30 days storage). The ranking of fried wedges (in the antioxidant administered oils) based on defuzzified scores after 40 days were: sesame $>$ mustard $>$ soybean (deep fry), mustard $>$ soybean $>$ sesame (shallow fry) and soybean $>$ mustard $>$ sesame (par-fry).

Keywords: fried potato wedges, fuzzy logic, sensory evaluation, shelf life, texture studies
\end{abstract}

Sensory evaluation is an integral parameter of food quality, which involves the use of all five human senses to interpret the quality of foods (STONE \& SiDEL, 2004). This is a subjective test conducted by a panel of judges with perception of expected sensory attributes of the food product and the responses lie in the interval $[0,1]$, i.e. within the range of rejection and acceptance, commonly in linguistic form, and are not unanimous (CHAKRABORTY et al., 2011). To eliminate subjectivity, fuzzy logic has been reported to be an effective tool in analyzing linguistic judgment. It also allows ranking of food products based on panelists' preferences for the quality attributes (CHAKRABORTY et al., 2011). In fuzzy system, linguistic variables (such as 'not satisfactory', 'fair', 'good') are used to determine relationships between independent variables (such as colour, aroma, and flavour) and dependent variables (acceptance and rejection) (SinIJA \& Mishra, 2011). The linguistic data obtained by subjective evaluation is correlated to accurate and exact data from objective evaluation (DAS, 2005). Defuzzification provides a numeric value for the sensory assessment of food samples for direct interpretation. However, application of fuzzy logic on sensory data does not completely rule out ambiguity in quality assessment. Hence, in the present work, we have substantiated the fuzzy logic scores with objective evaluation.

The primary objective of this study is to ascertain the shelf lives of fried potato wedges in deep, shallow, and par-fried soybean, sesame, and mustard oils (with administered antioxidants) by application of fuzzy logic and by objective evaluation of textural attributes of the wedges (using instrumental analysis). The secondary objective is to establish the most suitable frying mode in each of these oils by ranking the fried potato wedges.

\footnotetext{
* To whom correspondence should be addressed. Phone: 91-33-24146666 (2598); fax: 91-33-24146822; e-mail: pb@ftbe.jdvu.ac.in
} 


\section{Materials and methods}

\subsection{Materials}

Soybean, sesame, and mustard oils, potatoes - Solanum tuberosum (Jyoti variety), Al foil, and LDPE (low density polyethylene) packets were purchased from a local super market of Kolkata, India. An electric deep fat fryer of M/s Shiva Kitchen Appliances, Kolkata, India; BHT, citric acid, potassium sulphate, and aluminium silicate of M/s RFCL, New Delhi, India, and highly pure grade nitrogen of M/s BOC India Ltd., Kolkata, India were used for the study.

\subsection{Methods}

1.2.1. Formulation of critic acid based antioxidant, frying and storage of potato wedges. The potatoes were cut to dimensions $(7 \mathrm{~cm} \times 1 \mathrm{~cm} \times 1 \mathrm{~cm})$ and the formulation of citric acid based antioxidant (ATX), frying, packaging, and storage were carried out in accordance to our previous reports (GHosh et al., 2012; GHOSH \& BHATTACHARJEe, 2012), without repeated frying. Post frying, the potato wedges were packed in Al foil-LDPE and stored at $-18{ }^{\circ} \mathrm{C}$ for $10,20,30$, and 40 days.

1.2.2. Sensory evaluation of the fried potato wedges. Sensory evaluation was conducted by a 10-member trained panel between 10 a.m.-12 noon (RANGANNA, 1987). The panelists judged the quality attributes of the samples viz. colour (CL), flavour (FL), crispness (CS), homogeneity and doneness (HD), and aftertaste (AT) using a 5-point hedonic scale [1: not satisfactory $(\mathrm{N})$; 2: fair $(\mathrm{F}) ; 3$ : medium $(\mathrm{M})$; 4: good $(\mathrm{G})$; 5: excellent $(\mathrm{E})$ ]. They also gave their preferences for the relative importance of the quality attributes of the samples viz. not at all important (NI), somewhat important (SI), important (I), highly important (HI), and extremely important (EI).

1.2.3. Fuzzy logic application on sensory scores. The panel response was used to derive relationship between the quality attributes and the relative importance of the same using fuzzy logic. Sets of three numbers known as 'triplets' were used to denote triangular membership function distribution pattern of sensory scores (Fig. 1(a)). These triplets were: 'not satisfactory/not at all important' ( 0025$)$, 'fair/somewhat important' (25 2525$)$, 'medium/ important' (50 25 25), 'good/highly important' (75 25 25), 'excellent/extremely important' (100 25 0). These values for sensory scores and relative weightage (Table 1) were calculated in accordance to CHAKRABORTY and co-workers (2011). The fuzzified score (SO) shown in Table 2 was obtained using $\mathrm{T}_{\mathrm{w}}$ and triplets of 'colour' $\mathrm{T}_{\mathrm{s}}$ using the 'extended product rule' (DAS, 2005).

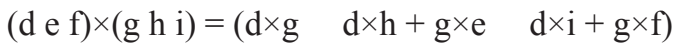

The fuzzified triplets were then added column-wise to obtain the overall fuzzified score $(\mathrm{S})$, in the form $(\mathrm{a} \mathrm{b} \mathrm{c})$ and then defuzzified using 'centre of gravity' method; the most common method of all defuzzification methods (LEE, 1990). Fig. 1(b) shows $\triangle \mathrm{ABC}$ representing the overall fuzzified triplet $(a b c)$. When the value of $(a+c)$ is $\leq 100$, the triangle ABC lies within the sensory scale interval $(0,100)$. The defuzzified score $(X)$ is (CHAKRABORTY et al., 2011): 


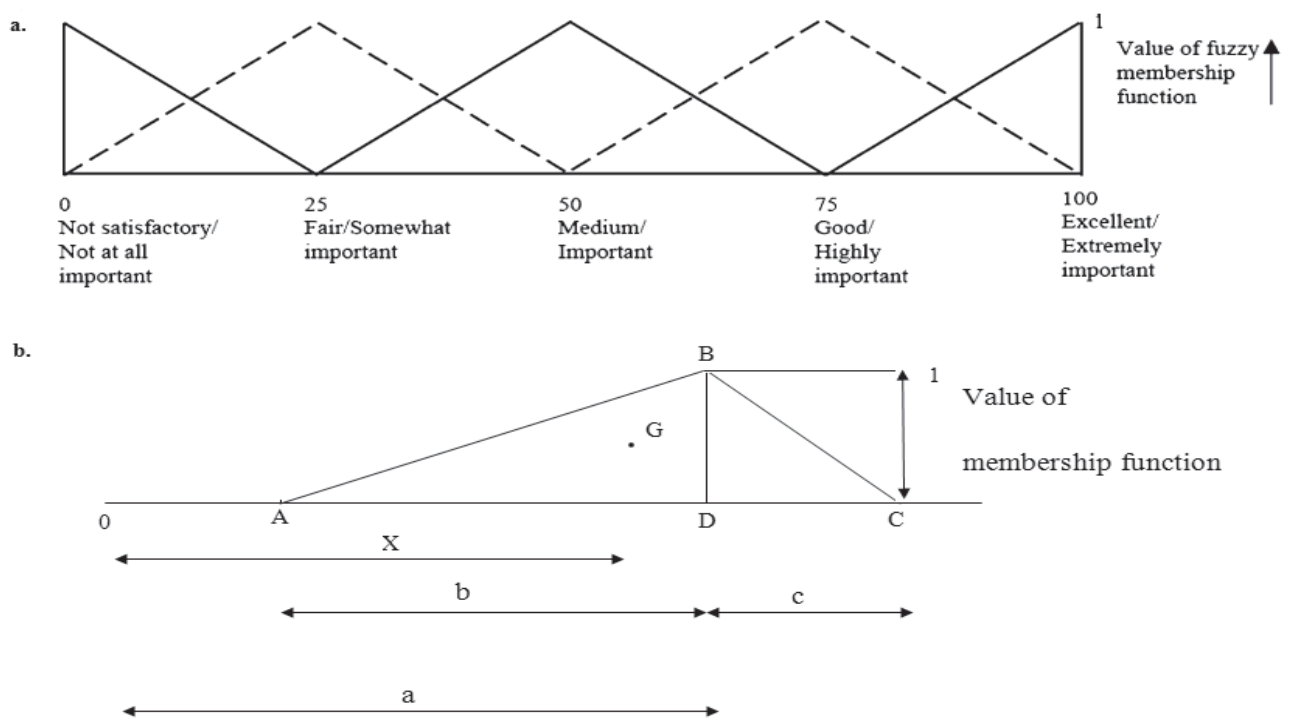

Fig. 1. Illustration of fuzzy logic by (a) triangular membership function of sensory scales and (b) graphical representation of overall fuzzified score $(a \mathrm{~b} c)$ and its membership function, $\mathrm{G}$ : centroid of the triangle $\mathrm{ABC}$; $\mathrm{X}$ : Defuzzified score

$$
\mathrm{X}=(3 \mathrm{a}-\mathrm{b}+\mathrm{c}) / 3
$$

All defuzzification calculations were conducted using Eq. 2. A defuzzified score of $\geq 50$ was considered as threshold for acceptance of quality attributes of fried potato wedges. The defuzzification of triplets for sensory scores $\left(\mathrm{T}_{\mathrm{s}}\right)$ was conducted, followed by ranking of quality attributes.

1.2.4. Texture analysis. The texture of the fried potato wedges were determined by Instron texture analyzer (M/s Instron Inc., Buckinghamshire, UK, model number 4301) with a $10 \mathrm{~N}$ load cell and $20 \mathrm{~mm} \mathrm{~min}^{-1}$ cross-head velocity, using a Warner-Bratzler shear blade. Hardness, shear modulus, and shear energy; the integral parameters in estimation of texture were measured (Mohan RAO \& Quintero, 2005). The experiments were conducted in triplicates and values were reported as mean $\pm \mathrm{SD}$ of three independent experiments (i.e., for three independent batches of fried potato wedges).

1.2.5. Statistical analysis. Statistical analysis was carried out by Student's $t$-test with 95\% confidence interval using STATISTICA 8.0 software (Statsoft, OK, USA).

\section{Results and discussion}

\subsection{Sensory analysis of fried potato wedges by panelists and application of fuzzy logic}

The panel judgment showed the ranking of the relative importance of the quality attributes of the fried wedges as: colour $>$ flavour $>$ crispness $>$ homogeneity and doneness $>$ aftertaste (Table 1). These results indicate that 'colour' was the most important criterion and 'aftertaste' the least, in agreement with common organoleptic experience of fried foods. 
The complete fuzzified and defuzzified scores are shown for deep fried potato wedges in soybean oil (control sample, 0 days storage) in Table 2 . Similar calculations were conducted for other batches as well (Table 3). The highest defuzzified scores (in ATX administered oils) were: 53.25 for par-fry soybean oil after 40 days of storage; 63.95 for deep fry sesame oil after 30 days of storage and a score of 56.94 for deep fry mustard oil after 30 days of storage. After 40 days of storage, we observed that the ranking of fried potato wedges (w.r.t. defuzzified scores) fried in ATX administered oils was: sesame oil $>$ mustard oil $>$ soybean oil for deep fry; mustard oil > soybean oil > sesame oil for shallow fry and soybean oil $>$ mustard oil $>$ sesame oil for par-fry (Tables 2 and 3). We observed that in all the batches, wedges fried in ATX administered oils were rated higher than their respective controls. Our results on efficiency of ATX were in agreement with WARNER and GEHRING (2009) on citric acid based antioxidant for improving shelf stability of fried foods. However, no literature on application of fuzzy logic on fried potato wedges is available to validate our study.

Table 1. Sum of number of panelists for relative importance of quality attributes of fried potato wedges and corresponding defuzzified values

\begin{tabular}{lcccccccccccccc}
\hline $\begin{array}{l}\text { Quality } \\
\text { attribute }\end{array}$ & $\mathrm{NI}^{\mathrm{a}}$ & $\mathrm{SI}^{\mathrm{a}}$ & $\mathrm{I}^{\mathrm{a}}$ & $\mathrm{HI}^{\mathrm{a}}$ & $\mathrm{EI}^{\mathrm{a}}$ & \multicolumn{3}{c}{$\mathrm{T}_{\mathrm{s}}$} & & & $\mathrm{T}_{\mathrm{w}}$ & & $\mathrm{X}^{\mathrm{b}}$ & Rank \\
\hline $\mathrm{CL}$ & 0 & 0 & 1 & 6 & 3 & 80.00 & 25.00 & 17.50 & 0.219 & 0.068 & 0.048 & 77.50 & $\mathrm{I}$ \\
FL & 0 & 0 & 1 & 7 & 2 & 77.50 & 25.00 & 20.00 & 0.212 & 0.068 & 0.055 & 75.83 & $\mathrm{II}$ & \\
CS & 0 & 0 & 1 & 8 & 1 & 75.00 & 25.00 & 22.50 & 0.205 & 0.068 & 0.062 & 74.16 & III \\
HD & 0 & 0 & 2 & 6 & 2 & 75.00 & 25.00 & 20.00 & 0.205 & 0.068 & 0.055 & 73.33 & IV \\
AT & 0 & 2 & 4 & 3 & 1 & 57.50 & 25.00 & 22.50 & 0.158 & 0.068 & 0.062 & 58.33 & V \\
\hline
\end{tabular}

${ }^{a}$ Number of panelists responding to a quality attribute of fried potato wedges, bdefuzzified values between 0-100, obtained by defuzzification of ' $\mathrm{T}_{\mathrm{s}}$ ' CL: colour; FL: flavour; CS: crispness; HD: homogeneity and doneness; AT: after taste; NI: not at all important; SI: somewhat important; I: important; HI: highly important; EI: extremely important; $\mathrm{T}_{\mathrm{s}}$ : triplets for sensory scores; $\mathrm{T}_{\mathrm{w}}$ : triplets for relative weightage; $\mathrm{X}$ : defuzzified sensory score of the relative importance of the quality attributes of the fried potato wedges

Table 2. Sum of number of panelists with different preferences for particular quality attributes of deep fried potato wedges in soybean oil for 0 days of storage and corresponding fuzzified and defuzzified values

\begin{tabular}{|c|c|c|c|c|c|c|c|c|c|c|c|c|c|c|c|c|c|}
\hline SD & ST & SP & $\mathrm{N}$ & $\mathrm{F}$ & $\mathrm{M}$ & G & $\mathrm{E}$ & & $\mathrm{T}_{\mathrm{s}}$ & & & $\mathrm{SO}$ & & & $\mathrm{S}$ & & $\mathrm{X}^{\mathrm{b}}$ \\
\hline \multirow[t]{5}{*}{0} & $\mathrm{CNT}$ & $\mathrm{CL}$ & 0 & 0 & 3 & 6 & 1 & 70.00 & 25.00 & 22.50 & 15.33 & 10.24 & 8.29 & & & & \\
\hline & & FL & 0 & 0 & 3 & 6 & 1 & 70.00 & 25.00 & 22.50 & 14.84 & 10.06 & 8.62 & & & & \\
\hline & & $\mathrm{CS}$ & 0 & 0 & 3 & 6 & 1 & 70.00 & 25.00 & 22.50 & 14.35 & 9.89 & 8.95 & 69.42 & 48.61 & 41.69 & 67.11 \\
\hline & & HD & 0 & 0 & 4 & 5 & 1 & 67.50 & 25.00 & 22.50 & 13.84 & 9.72 & 8.33 & & & & \\
\hline & & $\mathrm{AT}$ & 0 & 0 & 4 & 4 & 2 & 70.00 & 25.00 & 20.00 & 11.06 & 8.71 & 7.50 & & & & \\
\hline
\end{tabular}

b Defuzzified values between 0-100 obtained by defuzzification of 'S'. SD: storage days; ST: sample type; SP: sensory parameter; N: not satisfactory; F: fair; M: medium; G: good; E: excellent; CL: colour; FL: flavour; CS: crispness; HD: homogeneity and doneness; AT: aftertaste; $\mathrm{T}_{\mathrm{s}}$ : triplets for sensory scores; SO: fuzzified sensory score (triplets) of fried potato wedges; S: overall fuzzified sensory score (triplets) of the fried potato wedges; $\mathrm{X}$ : defuzzified sensory score of quality attributes of the fried potato wedges; CNT: samples fried incontrol oil; ATX: samples fried in antioxidant administered oil 
182 GHOSH \& BHATTACHARJEE: QUALITY ASSESSMENT OF POTATO WEDGES BY FUZZY LOGIC

Table 3. Defuzzified values ${ }^{\mathrm{b}}$ of fried potato wedges in soybean, sesame, and mustard oils with storage

\begin{tabular}{|c|c|c|c|c|c|c|c|c|c|c|}
\hline \multirow[t]{2}{*}{$\mathrm{SD}$} & \multirow[t]{2}{*}{ ST } & \multicolumn{3}{|c|}{ Soybean oil } & \multicolumn{3}{|c|}{ Sesame oil } & \multicolumn{3}{|c|}{ Mustard oil } \\
\hline & & Deep fry & $\begin{array}{c}\text { Shallow } \\
\text { fry }\end{array}$ & Par-fry & Deep fry & $\begin{array}{c}\text { Shallow } \\
\text { fry }\end{array}$ & Par-fry & Deep fry & $\begin{array}{c}\text { Shallow } \\
\text { fry }\end{array}$ & Par-fry \\
\hline \multirow[t]{2}{*}{0} & CNT & na & 70.64 & 73.07 & 70.19 & 63.56 & 65.14 & 73.77 & 71.41 & 75.30 \\
\hline & ATX & 72.00 & 72.43 & 74.43 & 72.92 & 70.11 & 72.12 & 75.85 & 76.90 & 76.57 \\
\hline \multirow[t]{2}{*}{10} & $\mathrm{CNT}$ & 66.79 & 55.60 & 72.85 & 67.86 & 52.46 & 56.95 & 68.38 & 62.17 & 51.47 \\
\hline & ATX & 70.50 & 61.74 & 73.04 & 72.00 & 67.54 & 57.45 & 71.92 & 70.29 & 57.73 \\
\hline \multirow[t]{2}{*}{20} & $\mathrm{CNT}$ & 53.49 & 42.14 & 67.94 & 51.95 & 42.47 & 44.78 & 60.86 & 52.72 & 46.68 \\
\hline & ATX & 58.54 & 58.25 & 72.28 & 70.96 & 61.27 & 47.50 & 69.86 & 62.19 & 53.64 \\
\hline \multirow[t]{2}{*}{30} & $\mathrm{CNT}$ & 51.83 & 41.30 & 52.62 & 48.69 & 40.01 & 35.62 & 44.14 & 43.90 & 43.08 \\
\hline & ATX & 53.99 & 56.71 & 65.14 & 63.95 & 48.84 & 43.08 & 56.94 & 48.58 & 48.95 \\
\hline \multirow[t]{2}{*}{40} & CNT & 38.38 & 36.59 & 41.75 & 39.02 & 30.67 & 29.28 & 40.53 & 40.54 & 39.40 \\
\hline & ATX & 45.12 & 45.33 & 53.25 & 48.84 & 43.40 & 38.56 & 47.91 & 45.92 & 45.58 \\
\hline
\end{tabular}

${ }^{\mathrm{b}}$ Defuzzified values between $0-100$.

SD: storage days; ST: sample type; CNT: samples fried in control oil; ATX: samples fried in antioxidant administered oil;

na: not applicable (data shown in Table 2)

\subsection{Texture analysis of the fried potato wedges}

From Table 4, highest values of hardness, shear modulus, and shear energy were obtained for deep fry, followed by shallow and par-fry. A significant difference $(\mathrm{P}<0.05)$ between wedges fried in ATX administered oils and control was observed for all samples, indicating better shelf stability of wedges fried in ATX administered oils. The minimum changes in values of 'hardness' were recorded for wedges fried in ATX administered oils with values of $2.2 \mathrm{~N}$ for soybean oil (40 days, parfry), followed by $2.5 \mathrm{~N}$ for mustard oil and $3.0 \mathrm{~N}$ for sesame oil (30 days, deep fry for both oils). Similarly, changes in shear modulus (ATX administered oils) were least for par-fry in soybean oil $\left(0.69 \times 10^{5} \mathrm{~Pa}\right)$ after 40 days, followed by that for deep fry mustard oil $\left(0.87 \times 10^{5} \mathrm{~Pa}\right)$ and deep fry sesame oil $\left(1.01 \times 10^{5} \mathrm{~Pa}\right)$, both after 30 days. The changes in shear energy (ATX administered oils) were lowest for par-fry soybean oil after 40 days of storage $\left(0.03 \mathrm{KJ} \mathrm{m}^{-3}\right)$, intermediate for deep fry sesame oil $\left(0.04 \mathrm{KJ} \mathrm{m}^{-3}\right)$ and highest for deep fry mustard oil $\left(0.05 \mathrm{KJ} \mathrm{m}^{-3}\right)$, both after 30 days.

The instrumental results agreed with sensory judgment and we obtained good correlation between hardness and defuzzified sensory scores (both in ATX administered oils) with correlation coefficient (r) values of $0.99,0.95$, and 0.96 for soybean oil; $0.90,0.93$, and 0.99 for sesame oil and $0.93,0.99$, and 0.92 for mustard oil, in deep, shallow, and par-fry, respectively. In our study, hardness, shear modulus, and shear energy values of the fried potato wedges have decreased with storage (Table 4), similar to sensory scores. Hence, from this study we affirm that texture profiling data are relevant alternatives of sensory scores. Our results were in agreement with sensory and textural studies reported by SEYMOUR and HAMANN (1988). 
GHOSH \& BHATTACHARJEE: QUALITY ASSESSMENT OF POTATO WEDGES BY FUZZY LOGIC 183

Table 4. Shear test results of hardness, shear modulus and shear energy of the fried potato wedges ${ }^{\mathrm{c}}$

\begin{tabular}{|c|c|c|c|c|c|c|c|c|}
\hline \multirow[t]{2}{*}{ Oil type } & \multirow[t]{2}{*}{ Fry type } & \multirow{2}{*}{$\begin{array}{c}\text { Storage } \\
\text { days }\end{array}$} & \multicolumn{2}{|c|}{ Hardness (N) } & \multicolumn{2}{|c|}{ Shear modulus $\left(10^{5} \mathrm{~Pa}\right)$} & \multicolumn{2}{|c|}{ Shear energy $\left(\mathrm{KJ} \mathrm{m}^{-3}\right)$} \\
\hline & & & CNT & ATX & CNT & ATX & CNT & ATX \\
\hline \multirow[t]{15}{*}{ Soybean } & Deep & 0 & $7.0 \pm 0.1^{\mathrm{d}}$ & $7.5 \pm 0.1^{\mathrm{e}}$ & $2.41 \pm 0.01^{\mathrm{d}}$ & $2.88 \pm 0.01^{\mathrm{e}}$ & $0.22 \pm 0.01^{\mathrm{d}}$ & $0.27 \pm 0.01^{\mathrm{e}}$ \\
\hline & & 10 & $6.8 \pm 0.1^{\mathrm{d}}$ & $6.9 \pm 0.1^{\mathrm{e}}$ & $1.65 \pm 0.01^{\mathrm{d}}$ & $2.56 \pm 0.01^{\mathrm{e}}$ & $0.22 \pm 0.02^{\mathrm{d}}$ & $0.25 \pm 0.01^{\mathrm{e}}$ \\
\hline & & 20 & $5.1 \pm 0.1^{\mathrm{d}}$ & $5.5 \pm 0.1^{\mathrm{e}}$ & $1.55 \pm 0.02^{\mathrm{d}}$ & $1.83 \pm 0.02^{\mathrm{e}}$ & $0.21 \pm 0.02^{\mathrm{d}}$ & $0.23 \pm 0.03^{\mathrm{e}}$ \\
\hline & & 30 & $4.3 \pm 0.1^{\mathrm{d}}$ & $4.8 \pm 0.2^{\mathrm{e}}$ & $0.91 \pm 0.01^{\mathrm{d}}$ & $1.12 \pm 0.02^{\mathrm{e}}$ & $0.20 \pm 0.01^{\mathrm{d}}$ & $0.22 \pm 0.01^{\mathrm{e}}$ \\
\hline & & 40 & $3.0 \pm 0.2^{\mathrm{d}}$ & $3.5 \pm 0.1^{\mathrm{e}}$ & $0.53 \pm 0.03^{\mathrm{d}}$ & $0.83 \pm 0.03^{\mathrm{e}}$ & $0.19 \pm 0.03^{\mathrm{d}}$ & $0.21 \pm 0.02^{\mathrm{e}}$ \\
\hline & Shallow & 0 & $6.3 \pm 0.1^{\mathrm{d}}$ & $6.6 \pm 0.1^{\mathrm{e}}$ & $1.75 \pm 0.01^{\mathrm{d}}$ & $1.94 \pm 0.01^{\mathrm{e}}$ & $0.21 \pm 0.01^{\mathrm{d}}$ & $0.26 \pm 0.01^{\mathrm{e}}$ \\
\hline & & 10 & $5.6 \pm 0.1^{\mathrm{d}}$ & $5.3 \pm 0.1^{\mathrm{e}}$ & $1.33 \pm 0.02^{\mathrm{d}}$ & $1.36 \pm 0.01^{\mathrm{e}}$ & $0.20 \pm 0.01^{\mathrm{d}}$ & $0.25 \pm 0.01^{\mathrm{e}}$ \\
\hline & & 20 & $4.8 \pm 0.1^{\mathrm{d}}$ & $5.1 \pm 0.1^{\mathrm{e}}$ & $1.04 \pm 0.01^{\mathrm{d}}$ & $1.19 \pm 0.01^{\mathrm{e}}$ & $0.19 \pm 0.02^{\mathrm{d}}$ & $0.24 \pm 0.01^{\mathrm{e}}$ \\
\hline & & 30 & $4.0 \pm 0.2^{\mathrm{d}}$ & $4.2 \pm 0.2^{\mathrm{e}}$ & $0.80 \pm 0.02^{\mathrm{d}}$ & $0.87 \pm 0.02^{\mathrm{e}}$ & $0.19 \pm 0.01^{\mathrm{d}}$ & $0.22 \pm 0.01^{\mathrm{e}}$ \\
\hline & & 40 & $2.3 \pm 0.1^{\mathrm{d}}$ & $2.8 \pm 0.1^{\mathrm{e}}$ & $0.41 \pm 0.03^{\mathrm{d}}$ & $0.54 \pm 0.02^{\mathrm{e}}$ & $0.18 \pm 0.03^{\mathrm{d}}$ & $0.20 \pm 0.01^{\mathrm{e}}$ \\
\hline & Par-fry & 0 & $5.0 \pm 0.1^{\mathrm{d}}$ & $5.0 \pm 0.1^{\mathrm{e}}$ & $1.16 \pm 0.01^{\mathrm{d}}$ & $1.19 \pm 0.01^{\mathrm{e}}$ & $0.19 \pm 0.01^{\mathrm{d}}$ & $0.23 \pm 0.01^{\mathrm{e}}$ \\
\hline & & 10 & $4.0 \pm 0.1^{\mathrm{d}}$ & $4.8 \pm 0.1^{\mathrm{e}}$ & $0.87 \pm 0.01^{\mathrm{d}}$ & $1.09 \pm 0.01^{\mathrm{e}}$ & $0.17 \pm 0.01^{\mathrm{d}}$ & $0.22 \pm 0.02^{\mathrm{e}}$ \\
\hline & & 20 & $3.5 \pm 0.1^{\mathrm{d}}$ & $4.4 \pm 0.2^{\mathrm{e}}$ & $0.67 \pm 0.02^{\mathrm{d}}$ & $0.92 \pm 0.02^{\mathrm{e}}$ & $0.17 \pm 0.02^{\mathrm{d}}$ & $0.21 \pm 0.01^{\mathrm{e}}$ \\
\hline & & 30 & $3.0 \pm 0.1^{\mathrm{d}}$ & $3.5 \pm 0.1^{\mathrm{e}}$ & $0.55 \pm 0.02^{\mathrm{d}}$ & $0.67 \pm 0.03^{\mathrm{e}}$ & $0.16 \pm 0.01^{\mathrm{d}}$ & $0.20 \pm 0.01^{\mathrm{e}}$ \\
\hline & & 40 & $2.4 \pm 0.2^{\mathrm{d}}$ & $2.8 \pm 0.1^{\mathrm{e}}$ & $0.41 \pm 0.01^{\mathrm{d}}$ & $0.50 \pm 0.01^{\mathrm{e}}$ & $0.14 \pm 0.01^{\mathrm{d}}$ & $0.20 \pm 0.01^{\mathrm{e}}$ \\
\hline \multirow[t]{15}{*}{ Sesame } & Deep & 0 & $7.0 \pm 0.2^{\mathrm{d}}$ & $8.5 \pm 0.1^{\mathrm{e}}$ & $2.80 \pm 0.02^{\mathrm{d}}$ & $3.54 \pm 0.01^{\mathrm{e}}$ & $0.23 \pm 0.02^{\mathrm{d}}$ & $0.30 \pm 0.02^{\mathrm{e}}$ \\
\hline & & 10 & $6.4 \pm 0.1^{\mathrm{d}}$ & $7.9 \pm 0.2^{\mathrm{e}}$ & $2.29 \pm 0.01^{\mathrm{d}}$ & $3.04 \pm 0.02^{\mathrm{e}}$ & $0.22 \pm 0.02^{\mathrm{d}}$ & $0.27 \pm 0.01^{\mathrm{e}}$ \\
\hline & & 20 & $5.8 \pm 0.2^{\mathrm{d}}$ & $7.2 \pm 0.1^{\mathrm{e}}$ & $1.71 \pm 0.03^{\mathrm{d}}$ & $2.80 \pm 0.01^{\mathrm{e}}$ & $0.21 \pm 0.01^{\mathrm{d}}$ & $0.26 \pm 0.02^{\mathrm{e}}$ \\
\hline & & 30 & $5.0 \pm 0.1^{\mathrm{d}}$ & $5.5 \pm 0.1^{\mathrm{e}}$ & $1.25 \pm 0.02^{\mathrm{d}}$ & $2.53 \pm 0.03^{\mathrm{e}}$ & $0.19 \pm 0.01^{\mathrm{d}}$ & $0.26 \pm 0.01^{\mathrm{e}}$ \\
\hline & & 40 & $2.8 \pm 0.4^{\mathrm{d}}$ & $4.8 \pm 0.4^{\mathrm{e}}$ & $0.62 \pm 0.03^{\mathrm{d}}$ & $1.17 \pm 0.02^{\mathrm{e}}$ & $0.17 \pm 0.01^{\mathrm{d}}$ & $0.20 \pm 0.02^{\mathrm{e}}$ \\
\hline & Shallow & 0 & $7.2 \pm 0.2^{\mathrm{d}}$ & $8.6 \pm 0.2^{\mathrm{e}}$ & $2.06 \pm 0.02^{\mathrm{d}}$ & $2.61 \pm 0.02^{\mathrm{e}}$ & $0.24 \pm 0.02^{\mathrm{d}}$ & $0.30 \pm 0.02^{\mathrm{e}}$ \\
\hline & & 10 & $6.2 \pm 0.1^{\mathrm{d}}$ & $7.2 \pm 0.1^{\mathrm{e}}$ & $1.63 \pm 0.01^{\mathrm{d}}$ & $2.12 \pm 0.02^{\mathrm{e}}$ & $0.24 \pm 0.01^{\mathrm{d}}$ & $0.28 \pm 0.01^{\mathrm{e}}$ \\
\hline & & 20 & $4.4 \pm 0.1^{\mathrm{d}}$ & $5.0 \pm 0.1^{\mathrm{e}}$ & $1.02 \pm 0.01^{\mathrm{d}}$ & $1.14 \pm 0.01^{\mathrm{e}}$ & $0.21 \pm 0.02^{\mathrm{d}}$ & $0.27 \pm 0.01^{\mathrm{e}}$ \\
\hline & & 30 & $3.8 \pm 0.2^{\mathrm{d}}$ & $4.4 \pm 0.2^{\mathrm{e}}$ & $0.72 \pm 0.04^{\mathrm{d}}$ & $0.89 \pm 0.03^{\mathrm{e}}$ & $0.21 \pm 0.02^{\mathrm{d}}$ & $0.25 \pm 0.01^{\mathrm{e}}$ \\
\hline & & 40 & $1.6 \pm 0.1^{\mathrm{d}}$ & $3.5 \pm 0.4^{\mathrm{e}}$ & $0.28 \pm 0.03^{\mathrm{d}}$ & $0.64 \pm 0.03^{\mathrm{e}}$ & $0.19 \pm 0.04^{\mathrm{d}}$ & $0.24 \pm 0.03^{\mathrm{e}}$ \\
\hline & Par-fry & 0 & $5.8 \pm 0.2^{\mathrm{d}}$ & $7.2 \pm 0.1^{\mathrm{e}}$ & $1.48 \pm 0.01^{\mathrm{d}}$ & $1.71 \pm 0.01^{\mathrm{e}}$ & $0.22 \pm 0.01^{\mathrm{d}}$ & $0.25 \pm 0.01^{\mathrm{e}}$ \\
\hline & & 10 & $4.2 \pm 0.2^{\mathrm{d}}$ & $5.3 \pm 0.2^{\mathrm{e}}$ & $0.86 \pm 0.02^{\mathrm{d}}$ & $1.15 \pm 0.02^{\mathrm{e}}$ & $0.21 \pm 0.01^{\mathrm{d}}$ & $0.24 \pm 0.01^{\mathrm{e}}$ \\
\hline & & 20 & $2.6 \pm 0.1^{\mathrm{d}}$ & $3.8 \pm 0.4^{\mathrm{e}}$ & $0.47 \pm 0.01^{\mathrm{d}}$ & $0.73 \pm 0.02^{\mathrm{e}}$ & $0.20 \pm 0.01^{\mathrm{d}}$ & $0.23 \pm 0.01^{\mathrm{e}}$ \\
\hline & & 30 & $1.8 \pm 0.4^{\mathrm{d}}$ & $2.3 \pm 0.5^{\mathrm{e}}$ & $0.29 \pm 0.02^{\mathrm{d}}$ & $0.38 \pm 0.02^{\mathrm{e}}$ & $0.19 \pm 0.02^{\mathrm{d}}$ & $0.21 \pm 0.01^{\mathrm{e}}$ \\
\hline & & 40 & $1.2 \pm 0.5^{\mathrm{d}}$ & $1.9 \pm 0.6^{\mathrm{e}}$ & $0.18 \pm 0.03^{\mathrm{d}}$ & $0.29 \pm 0.03^{\mathrm{e}}$ & $0.18 \pm 0.02^{\mathrm{d}}$ & $0.18 \pm 0.02^{\mathrm{d}}$ \\
\hline \multirow[t]{5}{*}{ Mustard } & Deep & 0 & $6.8 \pm 0.2^{\mathrm{d}}$ & $8.5 \pm 0.2^{\mathrm{e}}$ & $2.62 \pm 0.02^{\mathrm{d}}$ & $2.54 \pm 0.01^{\mathrm{e}}$ & $0.29 \pm 0.02^{\mathrm{d}}$ & $0.33 \pm 0.01^{\mathrm{e}}$ \\
\hline & & 10 & $6.1 \pm 0.1^{\mathrm{d}}$ & $7.1 \pm 0.3^{\mathrm{e}}$ & $2.03 \pm 0.01^{\mathrm{d}}$ & $2.54 \pm 0.02^{\mathrm{e}}$ & $0.27 \pm 0.02^{\mathrm{d}}$ & $0.32 \pm 0.01^{\mathrm{e}}$ \\
\hline & & 20 & $5.0 \pm 0.2^{\mathrm{d}}$ & $6.5 \pm 0.1^{\mathrm{e}}$ & $1.39 \pm 0.01^{\mathrm{d}}$ & $1.97 \pm 0.02^{\mathrm{e}}$ & $0.25 \pm 0.01^{\mathrm{d}}$ & $0.30 \pm 0.02^{\mathrm{e}}$ \\
\hline & & 30 & $3.4 \pm 0.1^{\mathrm{d}}$ & $6.0 \pm 0.5^{\mathrm{e}}$ & $0.85 \pm 0.03^{\mathrm{d}}$ & $1.67 \pm 0.03^{\mathrm{e}}$ & $0.22 \pm 0.02^{\mathrm{d}}$ & $0.28 \pm 0.02^{\mathrm{e}}$ \\
\hline & & 40 & $2.8 \pm 0.4^{\mathrm{d}}$ & $4.7 \pm 0.4^{\mathrm{e}}$ & $0.61 \pm 0.02^{\mathrm{d}}$ & $1.12 \pm 0.02^{\mathrm{e}}$ & $0.20 \pm 0.03^{\mathrm{d}}$ & $0.24 \pm 0.02^{\mathrm{e}}$ \\
\hline
\end{tabular}


Table 4. Continued

\begin{tabular}{|c|c|c|c|c|c|c|c|c|}
\hline \multirow[t]{2}{*}{ Oil type } & \multirow[t]{2}{*}{ Fry type } & \multirow{2}{*}{$\begin{array}{c}\text { Storage } \\
\text { days }\end{array}$} & \multicolumn{2}{|c|}{ Hardness $(\mathrm{N})$} & \multicolumn{2}{|c|}{ Shear modulus $\left(10^{5} \mathrm{~Pa}\right)$} & \multicolumn{2}{|c|}{ Shear energy $\left(\mathrm{KJ} \mathrm{m}^{-3}\right)$} \\
\hline & & & CNT & ATX & CNT & ATX & CNT & ATX \\
\hline & Shallow & 0 & $6.4 \pm 0.1^{\mathrm{d}}$ & $8.2 \pm 0.1^{\mathrm{e}}$ & $1.83 \pm 0.01^{\mathrm{d}}$ & $2.48 \pm 0.02^{\mathrm{e}}$ & $0.26 \pm 0.01^{\mathrm{d}}$ & $0.32 \pm 0.01^{\circ}$ \\
\hline & & 10 & $6.0 \pm 0.1^{\mathrm{d}}$ & $7.4 \pm 0.2^{\mathrm{e}}$ & $1.46 \pm 0.01^{\mathrm{d}}$ & $1.95 \pm 0.02^{\mathrm{e}}$ & $0.25 \pm 0.01^{\mathrm{d}}$ & $0.29 \pm 0.01^{\circ}$ \\
\hline & & 20 & $5.3 \pm 0.2^{\mathrm{d}}$ & $6.8 \pm 0.2^{\mathrm{e}}$ & $1.18 \pm 0.02^{\mathrm{d}}$ & $1.62 \pm 0.01^{\mathrm{e}}$ & $0.24 \pm 0.01^{\mathrm{d}}$ & $0.28 \pm 0.01^{\circ}$ \\
\hline & & 30 & $3.1 \pm 0.1^{\mathrm{d}}$ & $4.6 \pm 0.1^{\mathrm{e}}$ & $0.62 \pm 0.03^{\mathrm{d}}$ & $0.96 \pm 0.03^{\mathrm{e}}$ & $0.21 \pm 0.01^{\mathrm{d}}$ & $0.25 \pm 0.02$ \\
\hline & & 40 & $2.2 \pm 0.6^{\mathrm{d}}$ & $4.5 \pm 0.7^{\mathrm{e}}$ & $0.39 \pm 0.02^{\mathrm{d}}$ & $0.83 \pm 0.02^{\mathrm{e}}$ & $0.20 \pm 0.03^{\mathrm{d}}$ & $0.24 \pm 0.03^{\circ}$ \\
\hline & Par-fry & 0 & $5.2 \pm 0.2^{\mathrm{d}}$ & $7.2 \pm 0.2^{\mathrm{e}}$ & $1.16 \pm 0.01^{\mathrm{d}}$ & $1.67 \pm 0.01^{\mathrm{e}}$ & $0.23 \pm 0.02^{\mathrm{d}}$ & $0.26 \pm 0.01$ \\
\hline & & 10 & $4.6 \pm 0.1^{\mathrm{d}}$ & $6.1 \pm 0.1^{\mathrm{e}}$ & $0.88 \pm 0.02^{\mathrm{d}}$ & $1.27 \pm 0.01^{\mathrm{e}}$ & $0.22 \pm 0.01^{\mathrm{d}}$ & $0.25 \pm 0.02^{\circ}$ \\
\hline & & 20 & $4.0 \pm 0.3^{\mathrm{d}}$ & $4.8 \pm 0.2^{\mathrm{e}}$ & $0.67 \pm 0.02^{\mathrm{d}}$ & $0.86 \pm 0.02^{\mathrm{e}}$ & $0.20 \pm 0.02^{\mathrm{d}}$ & $0.24 \pm 0.01^{\circ}$ \\
\hline & & 30 & $1.8 \pm 0.3^{\mathrm{d}}$ & $3.2 \pm 0.1^{\mathrm{e}}$ & $0.28 \pm 0.03^{\mathrm{d}}$ & $0.53 \pm 0.02^{\mathrm{e}}$ & $0.19 \pm 0.01^{\mathrm{d}}$ & $0.22 \pm 0.01^{\circ}$ \\
\hline & & 40 & $1.2 \pm 0.6^{\mathrm{d}}$ & $2.7 \pm 0.4^{\mathrm{e}}$ & $0.18 \pm 0.04^{\mathrm{d}}$ & $0.42 \pm 0.03^{\mathrm{e}}$ & $0.18 \pm 0.02^{\mathrm{d}}$ & $0.20 \pm 0.02^{\circ}$ \\
\hline
\end{tabular}

${ }^{c}$ Values expressed are mean \pm standard deviation $(\mathrm{n}=3)$; d, different letters in the same row in a particular category indicate a significant difference $(\mathrm{P}<0.05)$. CNT: samples fried in control oil; ATX: samples fried in antioxidant administered oil

\section{Conclusions}

The best shelf life was obtained for potato wedges par-fried in soybean oil stored for 40 days and for those deep fried in sesame and mustard oils and stored for 30 days (using citric acid based antioxidant administered oils) by fuzzy logic and texture analyses. The most suitable frying modes for soybean, sesame, and mustard oils established by ranking the fried potato wedges (based on defuzzified scores after 40 days of storage) were: sesame $>$ mustard $>$ soybean (deep fry), mustard $>$ soybean $>$ sesame (shallow fry), and soybean $>$ mustard $>$ sesame (par-fry).

\section{References}

Chakraborty, D., Das, S. \& Das, H. (2011): Aggregation of sensory data using fuzzy logic of sensory quality evaluation of food. J. Fd Sci. Technol., doi:10.1007/s13197-011-0433-x.

DAs, H. (2005): Food processing operations analysis. 1st ed. Asian Books, New Delhi, India, pp. 383-389.

Ghosh, P.K., Chatterjee, D. \& Bhattacharjee, P. (2012): Alternative methods of frying and antioxidant stability in soybean oil. Adv. J. Fd Sci. Technol., 4, 26-33.

Ghosh, P.K. \& BhattacharJee, P. (2012): Alternative methods of frying and antioxidant stability in sesame and mustard oils. Acta Alimentaria, 42, 109-123.

LeE, C. (1990): Fuzzy logic in control systems: Fuzzy logic controller, Part I and II, IEEE T. Syst. Man Cyb., 20, 404-435.

Mohan Rao, V.N. \& Quintero, X. (2005): Rheological properties of solid foods. -in: Rao, M.A., Rizvi, S.S.H. \& DatTA, A.K. (Eds.) Engineering Properties of Foods. $3^{\text {rd }}$ ed. CRC Press, Boca Raton, FL, USA. pp. 101-145.

Ranganna, S. (1987): Handbook of analysis and quality control for fruit and vegetable products. $2^{\text {nd }}$ ed. Tata McGraw Hill, New Delhi, India. p. 597.

SiNIJA, V.R. \& MishrA, H.N. (2011): Fuzzy analysis of sensory data for quality evaluation and ranking of instant green tea powder and granules. Fd Bioprocess Technol., 4, 408-416.

Seymour, S.K. \& Hamann, D.D. (1988): Crispness and crunchiness of selected low moisture foods. J. Texture Stud., 19, 79-95.

Stone, H. \& Sidel, J.L. (2004): Sensory evaluation practices. $3^{\text {rd }}$ ed. Elsevier, CA, USA. p. 13.

WARNER, K. \& GeHring, M.M. (2009): High temperature natural antioxidant improves soy oil for frying. J. Fd Sci., $74,500-505$.

Acta Alimentaria 44, 2015 